\title{
LIFSHITZ TAILS FOR A CLASS OF SCHRÖDINGER OPERATORS WITH RANDOM BREATHER-TYPE POTENTIAL
}

\author{
WERNER KIRSCH AND IVAN VESELIĆ
}

\begin{abstract}
We derive bounds on the integrated density of states for a class of Schrödinger operators with a random potential. The potential depends on a sequence of random variables, not necessarily in a linear way. An example of such a random Schrödinger operator is the breather model, as introduced by Combes, Hislop and Mourre. For these models we show that the integrated density of states near the bottom of the spectrum behaves according to the so called Lifshitz asymptotics. This result can be used to prove Anderson localization in certain energy/disorder regimes.
\end{abstract}

\section{INTRODUCTION, MODEL AND RESUlT}

In this paper we study spectral properties of certain Schrödinger operators with random potential. The spectral theory of such operators has been studied since the eighties in the mathematical literature and there are several monographs devoted to this topic, see e.g. [7, $3,24,29]$. Certain spectral features, like the non-randomness of the spectral components and the integrated density of states, are shared by a wide variety of models under mild ergodicity and regularity assumptions. However specific characteristics - like the existence of a certain spectral type - depend on the concrete model at hand.

Our aim is to establish for a class of random Schrödinger operators the Lifshitz asymptotics of the integrated density of states (in the sequel abbreviated by IDS). Spectral edges at which the IDS exhibits Lifshitz tails are called fluctuation boundaries (of the spectrum). Based on physical intuition one expects that ergodic random Hamilton operators exhibit spectral localization in a neighbourhood of a fluctuation boundary. Here spectral localization means that in the relevant energy interval the spectrum is pure point, and the continuous spectral component is absent, almost surely. Rigorous proofs of localization oftentimes rely on the estimates on the finite volume approximation of the IDS implied by the Lifshitz asymptotics.

We define now the class of operators considered in this paper and thereafter present our results. They concern random Schrödinger operators $H_{\omega}$ of the following type

$$
H_{\omega}=H_{0}+V_{\text {per }}+V_{\omega}
$$

where $H_{0}=-\Delta$ denotes the Laplacian on $L^{2}\left(\mathbb{R}^{d}\right), V_{\text {per }}$ is a $\left(\mathbb{Z}^{d}-\right)$ periodic potential and $V_{\omega}$ is a random potential of the form

Date: 2010-08-12-breather.tex.

2000 Mathematics Subject Classification. 35J10; 82B44.

Key words and phrases. random Schrödinger operators, integrated density of states, Lifshitz tails, breather model, non-linear randomness.

(C) 2008 by the author. Faithful reproduction of this article, is permitted for non-commercial purposes. This work has been partially supported by the DFG within the Emmy-Noether-Project "Spectral properties of random Schrödinger operators and random operators on manifolds and graphs". 


$$
V_{\omega}(x):=\sum_{k \in \mathbb{Z}^{d}} u\left(\lambda_{k}(\omega), x-k\right) .
$$

Here $\lambda_{k}: \Omega \rightarrow\left[\lambda_{-}, \lambda_{+}\right], k \in \mathbb{Z}^{d}$ is a collection of non-trivial, independent, identically distributed random variables on the probability space $(\Omega, \mathcal{F}, \mathbb{P})$. The distribution of $\lambda_{0}$ is denoted by $\mu$ and we assume inf $\operatorname{supp} \mu=\lambda_{-}$. The function $u$ is called single site potential.

Throughout this paper we will make the following

\section{Assumptions:}

Periodic Potential: The potential $V_{\text {per }}$ is $\mathbb{Z}^{d}$-periodic and locally in $L^{p}\left(\mathbb{R}^{d}\right)$ for some $p>d$.

Random Potential: The single site potential $u: \mathbb{R} \times \mathbb{R}^{d} \rightarrow \mathbb{R}$ is jointly measurable and satisfies the following:

(i) For all $\lambda \in\left[\lambda_{-}, \lambda_{+}\right]$we have

$$
\operatorname{supp} u(\lambda, \cdot) \subset \Lambda_{1}:=\left[-\frac{1}{2}, \frac{1}{2}\right]^{d}
$$

(ii) We have for all $\lambda \in\left[\lambda_{-}, \lambda_{+}\right]$

$$
\frac{\partial}{\partial \lambda} u(\lambda, \cdot) \in L^{\infty}\left(\Lambda_{1}\right) \quad \text { with } \quad \kappa_{1}:=\sup _{x \in \mathbb{R}^{d}} \sup _{\lambda \in\left[\lambda_{-}, \lambda_{+}\right]}\left|\frac{\partial}{\partial \lambda} u(\lambda, x)\right|<\infty
$$

(iii) For all $x \in \mathbb{R}^{d}$ and $\lambda \in\left[\lambda_{-}, \lambda_{+}\right]$we have

$$
\frac{\partial u}{\partial \lambda}(\lambda, x) \geq 0
$$

(iv) There exist $\epsilon_{1}, \epsilon_{2}>0$ such that for all $\lambda \in\left[\lambda_{-}, \lambda_{-}+\epsilon_{2}\right]$ we have

$$
\frac{d}{d \lambda} \int_{\mathbb{R}^{d}} d x u(\lambda, x) \in\left[\epsilon_{1}, 1 / \epsilon_{1}\right]
$$

(v) There exist $\alpha, \kappa>0$ such that for all $\epsilon \leq \epsilon_{2}$

$$
\mu\left(\left[\lambda_{-}, \lambda_{-}+\epsilon\right)\right) \geq \alpha \epsilon^{\kappa}
$$

Note that due to the positivity assumption (ii) we have actually

$$
\kappa_{1}=\sup _{x \in \mathbb{R}^{d}} \sup _{\lambda \in\left[\lambda_{-}, \lambda_{+}\right]} \frac{\partial}{\partial \lambda} u(\lambda, x)
$$

A single site potential which stisfies conditions (i) - (iv) will be called monotone in the randomness. Note that the randomness enters the potential (2) via a field of random variables $\lambda_{k}, k \in \mathbb{Z}^{d}$, not necessarily in a linear way. A random potential of the form (2) with a single site potential satisfying the above Assumptions gives rise to a metrically transitive or ergodic operator, see e.g. [12] or [24] for the definition. This implies that there is a subset $\Sigma$ of the real line such that the spectrum of $H_{\omega}$ coincides with $\Sigma$ almost surely, and that there is a well defined IDS for the family $H_{\omega}, \omega \in \Omega$, see below for details.

Condition (iii) ensures that for all $\omega \in \Omega, x \in \mathbb{R}^{d}, k \in \mathbb{Z}^{d}$ we have

$$
u\left(\lambda_{k}(\omega), x\right) \geq u\left(\lambda_{-}, x\right)
$$


This enables us to write the potential part of the operator in a standardized way. If we set

$$
V_{0}(x):=\sum_{k \in \mathbb{Z}^{d}} u\left(\lambda_{-}, x-k\right) \quad \text { and } \quad \tilde{u}(\lambda, x):=u(\lambda, x)-u\left(\lambda_{-}, x\right)
$$

then $V_{0}$ is $\mathbb{Z}^{d}$-periodic and $\tilde{u}$ satisfies the same assumptions as the original single site potential $u$. Moreover $\tilde{u}(\lambda, x) \geq 0$ and $\tilde{u}\left(\lambda_{-}, x\right)=0$. Thus we may subsume $V_{0}$ into the potential $V_{\text {per }}$ using the relation $V_{\text {per }}+V_{\omega}=\left(V_{\text {per }}+V_{0}\right)+\left(V_{\omega}-V_{0}\right)$. Consequently, we may and will assume from now on without loss of generality that

$$
u(\lambda, x) \geq 0 \quad \text { and } \quad u\left(\lambda_{-}, x\right)=0 .
$$

It is easy to see that in this case $E_{0}:=\inf \sigma\left(H_{\text {per }}\right)$ equals inf $\sigma\left(H_{\omega}\right)$ almost surely.

Example 1. If we set in (2) $u(\lambda, x-k)=\lambda f(x-k)$ we obtain an alloy type potential

$$
V_{\omega}(x):=\sum_{k \in \mathbb{Z}^{d}} \lambda_{k}(\omega) f(x-k)
$$

Such random potentials have been thoroughly studied before in the context of the Lifshitz asymptotics of the integrated density of states and localization, see e.g. [14, 22, 16, 23, 4, 20, $13,18,28,8,29,10,11,1]$. If $f$ is non-negative and sufficiently regular the resulting single site potential $u$ is monotone in the randomness. For such alloy type models the results we are aiming at are by now well understood, therefore we will not elaborate on them further.

Example 2. The main example which motivated this paper was introduced in [5]. For this model we set

$$
u(\lambda, x)=-f(\lambda x)
$$

The resulting stochastic field

$$
V_{\omega}(x):=\sum_{k \in \mathbb{Z}^{d}}-f\left(\lambda_{k}(\omega)(x-k)\right)
$$

is called random breather-type potential, cf. $[5,6]$. If we assume for the function $f$ that

$$
\begin{array}{r}
\operatorname{supp} f \subset \Lambda_{\lambda_{-}}, \quad f \in C_{0}^{1}\left(\mathbb{R}^{d} \backslash\{0\}\right) \\
L^{\infty}\left(\mathbb{R}^{d}\right) \ni g(x):=-x \cdot(\nabla f)(x) \geq 0 \text { and not identically vanishing }
\end{array}
$$

then the potential $u: \mathbb{R} \times \mathbb{R}^{d} \rightarrow \mathbb{R}$ is monotone in the randomness. Inequality (8) is called the repulsivity property of $f$.

In order to formulate our main result we introduce some more notation. For a selfadjoint operator $A$ and a Borel set $J \subset \mathbb{R}$ we denote the associated spectral projection by $\chi_{J}(A)$. Let $\Lambda_{L}(j):=[-L / 2, L / 2]^{d}+j \subset \mathbb{R}^{d}$ be a cube of side length $L$ centered at $j \in \mathbb{Z}^{d}$. We write $\Lambda_{L}$ for $\Lambda_{L}(0)$ and denote by $\chi_{\Lambda_{L}}$ the characteristic function of this set. One possible way to define the IDS $N: \mathbb{R} \rightarrow \mathbb{R}$ is the following trace per unit volume formula

$$
N(E):=\mathbb{E}\left\{\operatorname{Tr}\left[\chi_{\Lambda_{1}} \chi_{]-\infty, E]}\left(H_{\omega}\right)\right]\right\}
$$

The almost sure infimum $E_{0}$ of the spectrum of the operator $H_{\omega}$ coincides with the energy $\inf \{E \in \mathbb{R} \mid N(E)>0\}$. For more details about the IDS we refer to the surveys [15] or [30] and references given there. 
The follwing result on the asymptotics of the IDS at the bottom of the spectrum tells us that it behaves roughly like $N(E) \sim \exp \left(-\right.$ const. $\left.\left(E-E_{0}\right)^{-d / 2}\right)$ for $E-E_{0}$ positive and small.

Theorem 3 (Lifshitz Tails). Let $H_{\omega}, \omega \in \Omega$ be a random operator with potential (2) satisfying the above assumptions. Then

$$
\lim _{E \searrow E_{0}} \frac{\log |\log N(E)|}{\log \left(E-E_{0}\right)}=-\frac{d}{2}
$$

This result can be used as a tool in a proof of spectral localization for breather-type models. In fact, the localization proof based on multiscale analysis usually requires two main ingredients, the Wegner estimate and an initial scale estimate. A Wegner estimate for breather-type models was given in [5] (see that paper for precise assumptions and [6] for related results). Those authors also prove localization under certain assumptions on the disorder and the energy. One can replace their initial scale estimate using Theorem 3 to obtain localization for small energies for breather-type models. We will not give details here.

Let us note that recently there has been a number of papers devoted to Lifshitz tails for models which depend non-monotonously on the randomness, cf. [2, 9, 21]. To treat such models one needs to use different methods than ours.

Let us make a comment on the difference between our proof of Lifshitz tails and the one for the standard alloy type model. The basic strategy of proof is the same, but since we are dealing here with a non-linear dependece on the randomness, we introduce a new family of (non-linearly) mapped random variables, which correspond to local energy contributions. Then we are in the position to make use of Temple's inequality similarly as in the case of alloy type potentials. To be able to control the relation of the second moment (of the energy) to the first moment we need to linearize it. This linearisation is one of the instances where we need the differentiability of the single site potentials with respect to the parameter $\lambda$ and sufficient control on the derivative.

As usual we prove Theorem 3 by giving an upper and a lower bound on $N$, respectively on the limit in Eq. (9). The next Section 2 is of preparatory nature where we discuss boundary conditions and corresponding bounds on the integrated density of states. The subsequent Section 3 contains the proof of the upper bound on the IDS. The lower bound is given in the final Section 4.

\section{BOUNDARY CONDITIONS}

In this section we discuss boundary conditions for operators on cubes $\Lambda_{L}$. For the upper bound on the IDS we will use operators $H_{0}^{L, \rho}$ on $L^{2}\left(\Lambda_{L}\right)$ with appropriate 'mixed' boundary conditions, formulated in terms of a bounded function $\rho$ on the boundary $\partial \Lambda_{L}$ of $\Lambda_{L}$. We say that a smooth function $\phi$ obeys the $\rho$-boundary conditions if $\rho \phi=-n \cdot \nabla \phi$ on $\partial \Lambda_{L}$. Here $n \cdot \nabla$ denotes the outer normal derivative at $\partial \Lambda_{L}$.

In a rigorous way we define $H_{0}^{L, \rho}$ as the operator associated to the sesquilinear form

$$
\left(\phi_{1}, \phi_{2}\right) \mapsto \int_{\Lambda_{L}} \overline{\nabla \phi_{1}(x)} \nabla \phi_{2}(x) d x+\int_{\partial \Lambda_{L}} \rho(x) \overline{\phi_{1}(x)} \phi_{2}(x) d x
$$

with the Sobolev space $\mathcal{H}^{1}\left(\Lambda_{L}\right)$ as its form domain. Here we use the same notation for a function $\phi$ on the cube $\Lambda_{L}$ and its trace on $\partial \Lambda_{L}$. Note that the second term of the sesquilinear 
form is well defined since the trace of $\phi$ is in $L^{2}\left(\partial \Lambda_{L}\right)$. The Neumann operator $H_{0}^{L, N}$ is given by the choice $\rho \equiv 0$, Dirichlet boundary conditions are formally given by $\rho \equiv \infty$. The Laplacian with Dirichlet boundary conditions $H_{0}^{L, D}$ is rigorously defined through the form

$$
\left(\phi_{1}, \phi_{2}\right) \mapsto \int_{\Lambda_{L}} \overline{\nabla \phi_{1}(x)} \nabla \phi_{2}(x) d x
$$

on $\mathcal{H}_{0}^{1}\left(\Lambda_{L}\right)$.

We will also need the operator $H_{0}^{L, P}$, the Hamiltonian with periodic boundary conditions at $\partial \Lambda_{L}$. Likewise we need notation for restrictions of the Schrödinger operators to finite cubes with selfadjoint boundary conditions. We set $H_{\mathrm{per}}^{L, \rho}=H_{0}^{L, \rho}+\chi_{\Lambda_{L}} V_{\mathrm{per}}, H_{\omega}^{L, \rho}=H_{0}^{L, \rho}+$ $\chi_{\Lambda_{L}} V_{\text {per }}+\chi_{\Lambda_{L}} V_{\omega}$ and similarly for Neumann, Dirichlet and periodic boundary conditions.

Next we discuss a special choice of mixed boundary conditions introduced by Mezincescu in [23]. For details see Mezincescu's paper or [19]. Denote by $\psi_{1}$ the $L^{2}$-normalized, positive ground state of $H_{\text {per }}^{1, P}$ and by $\Psi$ its periodic extension on the whole of $\mathbb{R}^{d}$. Then $\psi_{L}:=$ $L^{-d / 2} \chi_{\Lambda_{L}} \Psi$ is the normalized ground state of $H_{\text {per }}^{L, P}$. Since $\Psi$ is continuously differentiable and strictly positive (see e.g. [26]), we may define $\rho_{\Psi}(x):=-\frac{n \cdot \nabla \Psi(x)}{\Psi(x)}$. We will use the notaton $H_{\text {per }}^{L, M}=H_{\text {per }}^{L, \rho_{\Psi}}$ and $H_{\omega}^{L, M}=H_{\omega}^{L, \rho_{\Psi}}$ and refer to the corresponding boundary conditions as Mezincescu boundary conditions.

We denote the eigenvalues of the operator $H_{\omega}^{L, X}$ for $X \in\{D, N, M, P\}$ by

$$
E_{1}\left(H_{\omega}^{L, X}\right) \leq E_{2}\left(H_{\omega}^{L, X}\right) \leq \ldots \leq E_{n}\left(H_{\omega}^{L, X}\right) \leq \ldots
$$

with the convention that we repeat eigenvalues according to their multiplicity. We also define

$$
N\left(E, H_{\omega}^{L, X}\right)=\#\left\{n \mid E_{n}\left(H_{\omega}^{L, X}\right) \leq E\right\} .
$$

It is well known that the IDS can be obtained as a macroscopic limit of normalized eigenvalue counting functions

$$
N(E)=\lim _{L \rightarrow \infty} \frac{1}{L^{d}} N\left(E, H_{\omega}^{L, X}\right)
$$

for $X \in\{D, N, M, P\}$. The equality holds for almost all $\omega \in \Omega$ and all energies $E$ where the function $N$ is continuous. The following observation of Mezincescu (see [23] or [19]) will be crucial for our analysis:

$$
N(E)=\sup _{L \in \mathbb{N}} \frac{1}{L^{d}} \mathbb{E}\left(N\left(E, H_{\omega}^{L, D}\right)\right)=\inf _{L \in \mathbb{N}} \frac{1}{L^{d}} \mathbb{E}\left(N\left(E, H_{\omega}^{L, M}\right)\right)
$$

Equation (10) is a so called bracketing result. It allows us to estimate the integrated density of states from above using Mezincescu boundary conditions and from below using Dirichlet boundary conditions. Another important feature of Mezincescu boundary conditions is the relation

$$
E_{1}\left(H_{\text {per }}^{L, M}\right)=E_{1}\left(H_{\text {per }}^{L, P}\right)=\inf \sigma\left(H_{\text {per }}\right)=\inf \Sigma
$$

for all $L$. These relations follow from the following facts: Note that $\Psi$ is a positive distributional solution of $H_{\text {per }} \Psi=E_{1}\left(H_{\text {per }}^{L, P}\right) \Psi$ on all of $\mathbb{R}^{d}$. Thus the Allegretto-Piepenbrink Theorem tells us that $E_{1}\left(H_{\text {per }}^{L, P}\right) \leq \inf \sigma\left(H_{\text {per }}\right)$. On the other hand, by the Floquet-Bloch decomposition we know that $E_{1}\left(H_{\text {per }}^{L, P}\right)$ is inside of $\sigma\left(H_{\text {per }}\right)$, hence it is the infimum of this set. Moreover, the function $\psi_{L}$ is a normalized $L^{2}$-eigenfunction of $H_{\text {per }}^{L, M}$. Again by positivity it follows that it must be the ground state of this operator. Finally, note that since the 
random perturbation is monotone it follows that $\inf \sigma\left(H_{\text {per }}\right) \leq \inf \Sigma$. Standard arguments using sequences of approximate eigenfunctions (e.g. as in the proof of equation (1.1) in [18]) show that $\inf \sigma\left(H_{\text {per }}\right) \in \Sigma$.

Using the fact that $\psi_{L}$ is the normalized $L^{2}$-ground-state of $H_{\text {per }}^{L, M}$ Mezincescu generalizes in [23] the argument of [17] and deduces that there exists a constant $\epsilon_{0}>0$ such that

$$
E_{2}\left(H_{\text {per }}^{L, M}\right)-E_{1}\left(H_{\text {per }}^{L, M}\right) \geq \epsilon_{0} L^{-2}
$$

for all $L \in \mathbb{N}$.

\section{PRoOF OF THE UPPER BOUND}

In this section we prove the upper bound on the IDS. For simplicity of notation we will assume in the sequel that $E_{0}=\inf \sigma\left(H_{\text {per }}\right)=\inf \sigma\left(H_{\omega}\right)=0$. This can always be achieved by adding a constant to the periodic potential $V_{\text {per }}$. Our proof follows the strategy of [16], namely we will make use of (10) and of the positivity of $V_{\omega}$ to estimate for arbitrary $L \in \mathbb{N}$

$$
N(E) \leq \frac{1}{L^{d}} \mathbb{E}\left(N\left(E, H_{\omega}^{L, M}\right)\right) \leq \frac{1}{L^{d}} N\left(E, H_{\text {per }}^{L, M}\right) \mathbb{P}\left(E_{1}\left(H_{\omega}^{L, M}\right)<E\right)
$$

Since we are interested in the behavior of the IDS near the spectral bottom $E_{0}=0$ it will be sufficient to consider only energies $E \in[0,1]$. For $E \leq 1$ the quantity $\frac{1}{L^{d}} N\left(E, H_{\text {per }}^{L, M}\right)$ is bounded by a constant independet of $L \in \mathbb{N}, \omega \in \Omega$ and $E \in[0,1]$. So we are left with the task to bound $\mathbb{P}\left(E_{1}\left(H_{\omega}^{L, M}\right)<E\right)$ from above. To do so, we estimate $E_{1}\left(H_{\omega}^{L, M}\right)$ from below using Temple's inequality. We will find that for $E \sim L^{-2}$ we have

$$
\mathbb{P}\left(E_{1}\left(H_{\omega}^{L, M}\right)<E\right) \leq \exp \left(C L^{d}\right) \sim \exp \left(C^{\prime} E^{-d / 2}\right)
$$

which together with (12) gives the desired upper bound on $N$.

Now, we give the details of our proof, which is split into five steps. We start by introducing new random variables $\xi_{k}$ which correspont to local energy contributions.

Step 1 (Mapped random variables). On any compact set the function $\Psi$ is strictly positive by the Harnack inequality and bounded by subsolution estimates, see e.g. [7]. Since $\Psi$ is by definition periodic it is in fact uniformly bounded away from zero and from above,

$$
0<c_{3}:=\inf _{x \in \mathbb{R}^{d}} \Psi(x) \leq c_{4}:=\sup _{x \in \mathbb{R}^{d}} \Psi(x)<\infty
$$

We abbreviate by $d \alpha(x)$ the measure $\Psi(x)^{2} d x$ on $\mathbb{R}^{d}$.

We introduce for a parameter $c_{2} \leq \frac{\epsilon_{0} \epsilon_{1}}{2 c_{4}}$, i. e. $\frac{c_{2} c_{4}}{\epsilon_{1}} \leq \frac{\epsilon_{0}}{2}$, the cut-off random variables

$$
\tilde{\lambda}_{k}:=\min \left\{\lambda_{k}, \lambda_{-}+c_{2} L^{-2}\right\} \in\left[\lambda_{-}, \lambda_{-}+c_{2} L^{-2}\right]
$$

and the non-linearly mapped random variables

$$
\xi_{k}(\omega)=\xi\left(\tilde{\lambda}_{k}\right):=\int d \alpha(x) u\left(\tilde{\lambda}_{k}(\omega), x-k\right)
$$

Each $\xi_{k}$ corresponds to a summand $\left\langle\Psi, u\left(\tilde{\lambda}_{k}, \cdot-k\right) \Psi\right\rangle$ of the energy form depending on the random variable $\lambda_{k}$.

We derive two estimates which will be later needed for Temple's inequality. Set $I_{L}:=$ $\Lambda_{L} \cap \mathbb{Z}^{d}$ and denote $\tilde{H}_{\omega}:=H_{\text {per }}+\tilde{V}_{\omega}$ and $\tilde{V}_{\omega}:=\sum_{k \in \mathbb{Z}^{d}} u\left(\tilde{\lambda}_{k}(\omega), x-k\right)$. 
Step 2 (Analysis of the first moment). The following quadratic form will play a crucial role in the sequel. It may be understood as the first moment of the energy in the state $\psi_{L}$.

$$
\begin{aligned}
\left\langle\psi_{L}, \tilde{H}_{\omega}^{L, M} \psi_{L}\right\rangle=\left\langle\psi_{L}, H_{\mathrm{per}}^{L, M} \psi_{L}\right\rangle & +\left\langle\psi_{L}, \tilde{V}_{\omega} \psi_{L}\right\rangle=\left\langle\psi_{L}, \tilde{V}_{\omega} \psi_{L}\right\rangle \\
& =L^{-d} \sum_{k \in I_{L}} \int d \alpha(x) u\left(\tilde{\lambda}_{k}(\omega), x-k\right)=L^{-d} \sum_{k \in I_{L}} \xi_{k}(\omega)
\end{aligned}
$$

For $c_{2} L^{-2} \leq \epsilon_{2}$, i. e. $\tilde{\lambda}_{0} \leq \lambda_{-}+\epsilon_{2}$, we have

$$
\begin{aligned}
\xi\left(\tilde{\lambda}_{0}\right)=\int d \alpha(x) u\left(\tilde{\lambda}_{0}, x\right) & =\int_{\lambda_{-}}^{\tilde{\lambda}_{0}} d \tau \frac{d}{d \tau} \int d \alpha(x) u(\tau, x) \\
& \leq \frac{\tilde{\lambda}_{0}-\lambda_{-}}{\epsilon_{1}} c_{4} \quad \text { by Assumption (iv) } \\
& \leq \frac{c_{2}}{\epsilon_{1}} \frac{1}{L^{2}} c_{4} \quad \text { by definition of } \tilde{\lambda}_{0}
\end{aligned}
$$

Hence

$$
\left\langle\psi_{L}, \tilde{H}_{\omega}^{L, M} \psi_{L}\right\rangle=L^{-d} \sum_{k \in I_{L}} \xi_{k}(\omega) \leq \frac{c_{2} c_{4}}{\epsilon_{1}} \frac{1}{L^{2}}
$$

and thus for $\nu:=\frac{\epsilon_{0}}{2 L^{2}}+\left\langle\psi_{L}, \tilde{H}_{\omega}^{L, M} \psi_{L}\right\rangle$ we have

$$
\nu \leq \frac{\epsilon_{0}}{2} \frac{1}{L^{2}}+\frac{c_{2} c_{4}}{\epsilon_{1}} \frac{1}{L^{2}} \leq \frac{\epsilon_{0}}{L^{2}} \leq E_{2}\left(H_{\mathrm{per}}^{L, M}\right)
$$

by the choice of $c_{2}$, the normalization $E_{0}=0$, and Ineq. (11).

Step 3 (Analysis of the second moment). We will need also an estimate for the second moment. By the mean value theorem one sees that for some $\hat{\lambda} \in\left[\lambda_{-}, \lambda\right]$

$$
u^{2}(\lambda, x)=u^{2}(\lambda, x)-u^{2}\left(\lambda_{-}, x\right)=2\left(\lambda-\lambda_{-}\right) u(\hat{\lambda}, x) \frac{\partial u(\hat{\lambda}, x)}{\partial \hat{\lambda}}
$$

By Assumption (iii) we have $0 \leq u(\hat{\lambda}, x) \leq u(\lambda, x)$ and thus

$$
u^{2}(\lambda, x) \leq 2\left(\lambda-\lambda_{-}\right) u(\lambda, x) \frac{\partial u}{\partial \lambda}(\hat{\lambda}, x) \leq 2 \kappa_{1}\left(\lambda-\lambda_{-}\right) u(\lambda, x)
$$

by Assumption (ii). Hence

$$
\int d \alpha(x) u^{2}\left(\tilde{\lambda}_{k}, x-k\right) \leq 2 \kappa_{1} c_{2} L^{-2} \int d \alpha(x) u\left(\tilde{\lambda}_{k}, x-k\right)=2 \kappa_{1} c_{2} L^{-2} \xi_{k}
$$

and

$$
\left\|\tilde{H}_{\omega}^{L, M} \psi_{L}\right\|^{2}=L^{-d} \sum_{k \in I_{L}} \int d \alpha(x) u^{2}\left(\tilde{\lambda}_{k}, x-k\right) \leq 2 \kappa_{1} c_{2} L^{-2} L^{-d} \sum_{k \in I_{L}} \xi_{k}
$$

Step 4 (Lower bound for the first eigenvalue). The next theorem provides us with a lower bound on the first eigenvalue of a random box Hamiltonian. It is formulated in terms of an empirical average of the random variables $\xi_{k}$. To prove it we use Temple's inequality. The bounds on the first and second moment derived above are used on one hand to show that Temple's inequality is at all applicable, and on the other hand to insert them into the inequality to obtain an appropriate lower bound. 
Theorem 4. Choose $c_{2}$ small enough such that $c_{2} \leq \epsilon_{2} L^{2}$ and $4 \kappa_{1} c_{2} / \epsilon_{0}<1 / 4$. Then

$$
E_{1}\left(\tilde{H}_{\omega}^{L, M}\right) \geq \frac{3}{4} L^{-d} \sum_{k \in I_{L}} \xi_{k}(\omega)
$$

Proof. To ensure that Temple's inequality can be applied to the operator $\tilde{H}_{\omega}^{L, M}$ and the vector $\psi_{L}$, we need to establish a chain of inequalities, see for instance Theorem XIII.5 in [25]. Since $c_{2} L^{-2} \leq \epsilon_{2}$

$$
\begin{aligned}
0=E_{1}\left(H_{\mathrm{per}}^{L, M}\right) & \leq E_{1}\left(\tilde{H}_{\omega}^{L, M}\right) \\
& \leq\left\langle\psi_{L}, \tilde{H}_{\omega}^{L, M} \psi_{L}\right\rangle \\
& <\nu \\
& \leq E_{2}\left(H_{\mathrm{per}}^{L, M}\right) \\
& \leq E_{2}\left(\tilde{H}_{\omega}^{L, M}\right)
\end{aligned}
$$

by monotonicity (iii)

by the min-max Theorem

since $\epsilon_{0}>0$

by inequality (14)

by monotonicity (iii)

We have checked the prerequisites for Temple's inequality and may apply it to the operator $\tilde{H}_{\omega}^{L, M}$ and the vector $\psi_{L}$ :

$$
\begin{aligned}
E_{1}\left(\tilde{H}_{\omega}^{L, M}\right) & \geq\left\langle\psi_{L}, \tilde{H}_{\omega}^{L, M} \psi_{L}\right\rangle-\frac{\left\|\tilde{H}_{\omega}^{L, M} \psi_{L}\right\|^{2}}{\nu-\left\langle\psi_{L}, \tilde{H}_{\omega}^{L, M} \psi_{L}\right\rangle} \\
& \geq\left\langle\psi_{L}, \tilde{H}_{\omega}^{L, M} \psi_{L}\right\rangle-\frac{2 \kappa_{1} c_{2} L^{-2} L^{-d} \sum_{k \in I_{L}} \xi_{k}(\omega)}{\frac{\epsilon_{0}}{2} L^{-2}} \\
& \geq\left\langle\psi_{L}, \tilde{H}_{\omega}^{L, M} \psi_{L}\right\rangle-\frac{4 \kappa_{1} c_{2}}{\epsilon_{0}} L^{-d} \sum_{k \in I_{L}} \xi_{k}(\omega)
\end{aligned}
$$

Here we used equation (15). It follows from equation (13) that

$$
E_{1}\left(\tilde{H}_{\omega}^{L, M}\right) \geq\left(1-\frac{4 \kappa_{1} c_{2}}{\epsilon_{0}}\right)\left\langle\psi_{L}, \tilde{H}_{\omega}^{L, M} \psi_{L}\right\rangle=\left(1-\frac{4 \kappa_{1} c_{2}}{\epsilon_{0}}\right) \frac{1}{L^{d}} \sum_{k \in I_{L}} \xi_{k}(\omega)
$$

and thus we have proven the Theorem.

The theorem in turn implies an estimate on how small most of the random variables $\xi_{k}, k \in I_{L}$ must be, if the principal eigenvalue of $\tilde{H}_{\omega}^{L}$ is low.

Corollary 5. Let $4 \kappa_{1} c_{2} / \epsilon_{0}<1 / 4$ and $\gamma>1$. Then we have

$$
E_{1}\left(\tilde{H}_{\omega}^{L, M}\right) \leq \mathcal{E} \text { implies } \#\left\{k \in I_{L} \mid \xi_{k}<2 \gamma \mathcal{E}\right\}>\frac{\gamma-1}{\gamma} L^{d}
$$

Proof. If the conclusion is false then

$$
\#\left\{k \in I_{L} \mid \xi_{k} \geq 2 \gamma \mathcal{E}\right\} \geq \frac{L^{d}}{\gamma}
$$

Hence $\sum_{k \in I_{L}} \xi_{k}(\omega) \geq 2 \gamma \mathcal{E} \frac{L^{d}}{\gamma}=2 \mathcal{E} L^{d}$. Theorem 4 implies

$$
E_{1}\left(\tilde{H}_{\omega}^{L, M}\right) \geq \frac{3}{4} L^{-d} \cdot 2 \mathcal{E} L^{d}=\frac{3}{2} \mathcal{E}>E_{1}\left(\tilde{H}_{\omega}^{L, M}\right)
$$

which yields a contradiction. 
Step 5 (Large deviation estimate). Now we have to show that the event

$$
\#\left\{k \in I_{L} \mid \xi_{k}<2 \gamma \mathcal{E}\right\}>\frac{\gamma-1}{\gamma} L^{d}
$$

has an exponentially small probability in the parameter $L^{d}$. To this aim we transform back first to the random variables $\tilde{\lambda}_{k}, k \in I_{L}$ and then to $\lambda_{k}, k \in I_{L}$.

Lemma 6. For $\frac{2 \gamma}{\epsilon_{1} c_{3}^{2}} \leq c_{7} \leq \epsilon_{2} / \mathcal{E}$, we have

$$
\xi_{k} \in\left[0,2 \gamma \mathcal{E}\left[\text { implies } \tilde { \lambda } _ { k } \in \left[\lambda_{-}, \lambda_{-}+c_{7} \mathcal{E}[\right.\right.\right.
$$

Proof. Assume $\tilde{\lambda}_{k} \geq \lambda_{-}+c_{7} \mathcal{E}$. By (iii) and (vi) we have for $c_{7} \mathcal{E} \leq \epsilon_{2}$

$$
\begin{aligned}
\xi_{k}(\omega) & \geq \int d \alpha(x) u\left(\lambda_{-}+c_{7} \mathcal{E}, x\right) \\
& \geq c_{3}^{2} \int_{\lambda_{-}}^{\lambda_{-}+c_{7} \mathcal{E}} d \tau \frac{d}{d \tau} \int d x u(\tau, x) \geq c_{3}^{2} c_{7} \mathcal{E} \epsilon_{1}
\end{aligned}
$$

Since $c_{7} \geq \frac{2 \gamma}{\epsilon_{1} c_{3}^{2}}$, it follows $\xi_{k} \geq 2 \gamma \mathcal{E}$ which is a contradiction.

Choose $c_{7} \mathcal{E} \leq \frac{c_{2}}{2 L^{2}}$. Then $\tilde{\lambda}_{k} \in\left[\lambda_{-}, \lambda_{-}+c_{7} \mathcal{E}\left[\right.\right.$ implies $\lambda_{k} \in\left[\lambda_{-}, \lambda_{-}+c_{7} \mathcal{E}[\right.$. Thus we have shown that for $c_{7} \geq \frac{2 \gamma}{\epsilon_{1} c_{3}^{2}}$ and $c_{7} \mathcal{E} \leq \min \left(\epsilon_{2}, \frac{c_{2}}{2 L^{2}}\right)$

$$
E_{1}\left(\tilde{H}_{\omega}^{L, M}\right) \leq \mathcal{E} \quad \text { implies } \quad \#\left\{k \in I_{L} \mid \lambda_{k}<\lambda_{-}+c_{7} \mathcal{E}\right\}>\frac{\gamma-1}{\gamma} L^{d}
$$

Since $\mu\left(\left\{\lambda_{-}\right\}\right)<1$ there exists a $\left.\lambda_{*} \in\right] \lambda_{-}, \lambda_{+}\left[\right.$such that $\left.p:=\mu\left(\left[\lambda_{*}, \lambda_{+}\right]\right) \in\right] 0,1[$. For $L$ large enough we have $\lambda_{-}+c_{7} \mathcal{E} \leq \lambda_{-}+\frac{c_{2}}{2 L^{2}} \leq \lambda_{*}$, hence

$$
\begin{aligned}
\mathbb{P}\left(\#\left\{k \in I_{L} \mid \lambda_{k}<\lambda_{-}+c_{7} \mathcal{E}\right\}>\frac{\gamma-1}{\gamma} L^{d}\right) & \leq \mathbb{P}\left(\#\left\{k \in I_{L} \mid \lambda_{k}<\lambda_{*}\right\}>\frac{\gamma-1}{\gamma} L^{d}\right) \\
& =\mathbb{P}\left(\#\left\{k \in I_{L} \mid \lambda_{k} \geq \lambda_{*}\right\}<\frac{1}{\gamma} L^{d}\right)
\end{aligned}
$$

The latter probability is bounded by $\exp \left(-\frac{1}{2} p^{2} L^{d}\right)$ if we choose $\gamma=2 / p$, cf. Theorem 4.2 in [27]. To conclude the proof of the upper bound we specify the choice

$$
L:=\left\lfloor\sqrt{\frac{c_{2}}{2 c_{7} \mathcal{E}}}\right\rfloor
$$

\section{Proof of the LOWER BOUnd}

Now we derive a lower bound on the IDS for energies above, but close to $E_{0}=0$. For this purpose we deduce from estimate (10) and the Čebyšev inequality

$$
N(E) \geq \frac{1}{L^{d}} \mathbb{E}\left(N\left(E, H_{\omega}^{L, D}\right)\right) \geq \frac{1}{L^{d}} \mathbb{P}\left(E_{1}\left(H_{\omega}^{L, D}\right) \leq E\right)
$$

To bound $E_{1}\left(H_{\omega}^{L, D}\right)$ from above we use the following Lemma which can be found in [16] and in $[19]$. 
Lemma 7. There are constants $B_{1}, B_{2} \in[0, \infty[$ such that

$$
E_{1}\left(H_{\omega}^{L, D}\right) \leq B_{1} \frac{1}{L^{d}} \int_{\Lambda_{L}} V_{\omega}(x) d x+B_{2} L^{-2}
$$

for all $\omega \in \Omega$ and $L \in \mathbb{N}$.

Due to Assumption (iv) we know that for $\lambda_{-} \leq \lambda \leq \lambda_{-}+\epsilon_{2}$ we have $\int_{\mathbb{R}^{d}} u(\lambda, x) d x \leq$ $\left(\lambda-\lambda_{-}\right) / \epsilon_{1}$. So, if $\lambda_{k}-\lambda_{-} \leq \delta$ for all $k \in I_{L}$ with $\delta$ small enough, then

$$
\frac{1}{L^{d}} \int_{\Lambda_{L}} V_{\omega}(x) d x \leq \frac{1}{L^{d}} \sum_{k \in I_{L}} \frac{\lambda_{k}(\omega)-\lambda_{-}}{\epsilon_{1}} \leq \frac{\delta}{\epsilon_{1}}
$$

and consequently $E_{1}\left(H_{\omega}^{L, D}\right) \leq B_{1} \frac{\delta}{\epsilon_{1}}+B_{2} L^{-2} \leq \frac{3}{4} E$ if we choose $\delta=\frac{\epsilon_{1}}{2 B_{1}} E$ and $L:=$ $\left\lceil 2 \sqrt{B_{2}} E^{-1 / 2}\right\rceil$. Combining these estimates we obtain

$$
\begin{aligned}
N(E) & \geq \frac{1}{L^{d}} \mathbb{P}\left(\lambda_{k}-\lambda_{-} \leq \frac{\epsilon_{1}}{2 B_{1}} E ; \text { for all } k \in I_{L}\right) \\
& \geq \frac{1}{L^{d}} \mathbb{P}\left(\lambda_{0}-\lambda_{-} \leq \frac{\epsilon_{1}}{2 B_{1}} E\right)^{L^{d}} \geq \frac{1}{L^{d}} \alpha^{\prime} E^{\kappa L^{d}}
\end{aligned}
$$

by Assumption (v), where $\alpha^{\prime}=\alpha\left(\frac{\epsilon_{1} E}{2 B_{1}}\right)^{\kappa L^{d}}$. Since for $E$ small $L^{d} \leq\left(4^{d} B_{2}^{d / 2}\right) E^{-d / 2}$, it follows that

$$
\lim _{E \searrow 0} \frac{\log |\log N(E)|}{\log E} \geq-\frac{d}{2}
$$

Acknowledgements

We are grateful to an anonymous referee for careful reading of the manuscript and valuable comments.

\section{REFERENCES}

[1] M. Aizenman, A. Elgart, S. Naboko, J. H. Schenker, and G. Stolz. Moment analysis for localization in random Schrödinger operators. Invent. Math., 163(2):343-413, 2006.

[2] J. Baker, M. Loss, and G. Stolz. Minimizing the ground state energy of an electron in a randomly deformed lattice. Comm. Math. Phys., 283(2):397-415, 2008.

[3] R. Carmona and J. Lacroix. Spectral Theory of Random Schrödinger Operators. Birkhäuser, Boston, 1990.

[4] J.-M. Combes and P. Hislop. Localization for some continuous, random Hamiltionians in $d$-dimensions. $J$. Funct. Anal., 124:149-180, 1994.

[5] J.-M. Combes, P. D. Hislop, and E. Mourre. Spectral averaging, perturbation of singular spectra, and localization. Trans. Amer. Math. Soc., 348(12):4883-4894, 1996.

[6] J.-M. Combes, P. D. Hislop, and S. Nakamura. The $L^{p}$-theory of the spectral shift function, the Wegner estimate, and the integrated density of states for some random Schrödinger operators. Commun. Math. Phys., 70(218):113-130, 2001.

[7] H. L. Cycon, R. G. Froese, W. Kirsch, and B. Simon. Schrödinger Operators with Application to Quantum Mechanics and Global Geometry. Text and Monographs in Physics. Springer, Berlin, 1987.

[8] D. Damanik and P. Stollmann. Multi-scale analysis implies strong dynamical localization. Geom. Funct. Anal., 11(1):11-29, 2001.

[9] R. Fukushima. Brownian survival and Lifshitz tail in perturbed lattice disorder. J. Funct. Anal., 256(9):2867-2893, 2009.

[10] F. Germinet and A. Klein. Bootstrap multiscale analysis and localization in random media. Comm. Math. Phys., 222(2):415-448, 2001.

[11] F. Germinet and A. Klein. A characterization of the Anderson metal-insulator transport transition. Duke Math. J., 124(2):309-350, 2004. 
[12] W. Kirsch. Random Schrödinger operators. In H. Holden and A. Jensen, editors, Schrödinger Operators, Lecture Notes in Physics, 345, Berlin, 1989. Springer.

[13] W. Kirsch. Wegner estimates and Anderson localization for alloy-type potentials. Math. Z., 221:507-512, 1996.

[14] W. Kirsch and F. Martinelli. Large deviations and Lifshitz singularity of the integrated density of states of random Hamitonians. Commun. Math. Phys., 89:27-40, 1983.

[15] W. Kirsch and B. Metzger. The integrated density of states for random Schrödinger operators. In Spectral Theory and Mathematical Physics, volume 76 of Proceedings of Symposia in Pure Mathematics, pages 649-698. AMS, 2007.

[16] W. Kirsch and B. Simon. Lifshitz tails for periodic plus random potentials. J. Stat. Phys., 42:799-808, 1986.

[17] W. Kirsch and B. Simon. Comparison theorems for the gap of Schrödinger operators. J. Funct. Anal., 75:396-410, 1987.

[18] W. Kirsch, P. Stollmann, and G. Stolz. Localization for random perturbations of periodic Schrödinger operators. Random Oper. Stochastic Equations, 6(3):241-268, 1998.

[19] W. Kirsch and S. Warzel. Lifshits tails caused by anisotropic decay: the emergence of a quantum-classical regime. Math. Phys. Anal. Geom., 8(3):257-285, 2005.

[20] F. Klopp. Localization for some continuous random Schrödinger operators. Commun. Math. Phys., 167:553-569, 1995.

[21] F. Klopp and S. Nakamura. Spectral extrema and Lifshitz tails for non-monotonous alloy type models. Comm. Math. Phys., 287(3):1133-1143, 2009.

[22] F. Martinelli and H. Holden. On absence of diffusion near the bottom of the spectrum for a random Schrödinger operator on $L^{2}\left(R^{\nu}\right)$. Commun. Math. Phys., 93:197-217, 1984.

[23] G. A. Mezincescu. Lifschitz singularities for periodic operators plus random potentials. J. Statist. Phys., 49(5-6):1181-1190, 1987.

[24] L. A. Pastur and A. L. Figotin. Spectra of Random and Almost-Periodic Operators. Springer, Berlin, 1992.

[25] M. Reed and B. Simon. Methods of Modern Mathematical Physics IV, Analysis of Operators. Academic Press, San Diego, 1978.

[26] B. Simon. Schrödinger Semigroups. Bull. Am. Math. Soc., 7:447-526, 1982.

[27] B. Simon. Lifschitz tails for the Anderson model. J. Stat. Phys., 38:65-76, 1985.

[28] P. Stollmann. Lifshitz asymptotics via linear coupling of disorder. Math. Phys. Anal. Geom., 2(3):279-289, 1999.

[29] P. Stollmann. Caught by disorder: Bound States in Random Media, volume 20 of Progress in Mathematical Physics. Birkhäuser, 2001.

[30] I. Veselić. Existence and regularity properties of the integrated density of states of random Schrödinger Operators, volume Vol. 1917 of Lecture Notes in Mathematics. Springer, 2007.

(W. K.) Fakultät für Mathematik und Informatik, FernUniversität Hagen, Germany

$U R L:$ www. fernuni-hagen.de/WTHEORIE/

(I. V.) Fakultät für Mathematik, 09107 TU-Chemnitz, Germany

URL: http://www.tu-chemnitz.de/mathematik/stochastik 